\title{
PÉ DIABÉTICO: FATORES DE RISCO PARA AMPUTAÇÃo
}

\author{
Vitória Almeida Matos da Silva ${ }^{1}$; Ana Mayra Andrade de Oliveira ${ }^{2}$; Aline da Silva Santos \\ Silva $^{3}$; Gabriela Haana Maia dos Santos Oliveira ${ }^{4}, L$ Ĺlia Paula de Souza Santos ${ }^{5}$ \\ 1.Bolsista PIBIC/FAPESB, Graduanda em Enfermagem, Universidade Estadual de Feira de Santana. \\ Email: vitoriauefs@gmail.com \\ 2.Professora Doutora e Orientadora do Núcleo de Pesquisa de Endocrinologia, Departamento de Saúde, \\ Universidade Estadual de Feira de Santana. Email: anamayra@uol.com.br \\ 3. Bolsista PROBIC/UEFS, Graduanda em Medicina, Universidade Estadual de Feira de Santana. \\ Email: aliness1612@gmail.com \\ 4. Bolsista PROBIC/UEFS, Graduanda em Medicina, Universidade Estadual de Feira de Santana. \\ Email: gabihaana@hotmail.com \\ 5. Mestre em Saúde Coletiva, Universidade Estadual de Feira de Santana. \\ Email: lipss13@hotmail.com.br
}

PALAVRAS-CHAVE: Pé Diabético, Amputação, Fatores de Risco

\section{INTRODUÇÃO}

O diabetes mellitus (DM) caracteriza-se por um grupo heterogêneo de distúrbios metabólicos que apresenta em comum a hiperglicemia crônica, resultado da deficiência na ação e/ou secreção de insulina (DIRETRIZES SBD, 2016).

Estima-se que em todo o mundo 1 em 11 adultos tem diabetes (415 milhões) e em 2040, 1 adulto em cada 10 terá diabetes, resultando num total de 642 milhões (IDF, 2017 ). E, que A cada 6 segundos, uma pessoa morre de diabetes (5,0 milhões de mortes) (IDF, 2017). Esse aumento global da prevalência de diabetes tem elevado dramaticamente o número de complicações crônicas da doença que apresentam elevada morbimortalidade (Wild et al, 2004).

As complicações crônicas do DM se dividem em micro e macrovasculares, sendo uma das complicações mais importantes, o Pé Diabético (PD), que segundo o Grupo de Trabalho Internacional sobre Pé Diabético ou International Working Group on the Diabetic Foot é conceituado como a presença de infecção, ulceração e/ou destruição de tecidos moles associados a alterações neurológicas e a vários graus de doença arterial periférica (DAP) nos membros inferiores, que pode culminar em amputação, portanto, uma complicação com elevado custo individual, social e econômico.

A incidência cumulativa de ulceração ao longo da vida entre pacientes com DM é estimada em $25,4 \%$ ressaltando-se que $85 \%$ das úlceras precedem as amputações (DIRETRIZES SBD, 2016). Estima-se que a cada 20 segundos uma pessoa sofre amputação de membro inferior decorrente do DM em todo o mundo, e que até $85 \%$ destes procedimentos, pode ser prevenido com uma triagem adequada (Apelqvist et al, 2008).

Tais aspectos epidemiológicos justificam a realização de estudos que visem analisar fatores de risco para amputações em pacientes com DM, bem como a disponibilidade de serviços de reabilitação de indivíduos amputados. Uma vez que tais dados podem fornecer a base para futuras intervenções em populações caracterizadas como de alto risco.

\section{METODOLOGIA}

Trata-se de estudo de corte transversal, quantitativo e do tipo exploratório. Esta pesquisa foi realizada na cidade de Feira de Santana (BA), com indivíduos provenientes do ambulatório do Centro de Atendimento ao Diabético e Hipertenso (CADH). Foram incluídos todos os pacientes com diagnóstico de DM e PD de acordo com os critérios da Sociedade 
Brasileira de Diabetes (2016), apresentando mais de 10 anos de doença (classificados de acordo com o GTIPD - Grupo de Trabalho Internacional sobre Pé Diabético , ano). Foram excluídas gestantes, e pessoas que sofreram revascularização cardíaca ou de membros inferiores no último ano.

A coleta de dados foi realizada através da utilização de ficha contendo informações epidemiológicas e clínicas dos portadores de DM (Tempo de doença, neuropatia, hábitos de vida, doenças cardiovasculares, nefropatias etc ) . Assim como, sobre o seu conhecimento a respeito dos cuidados com os seus pés, tipo de calçado comumente usado, sobre a disponibilidade dos serviços quanto à dispensação gratuita de órteses, calçados e próteses. E também a respeito da avaliação de estratégias educacionais referentes ao cuidado com o PD realizadas na Unidade de Saúde. Ao final foi realizada uma reunião na Unidade de Saúde, com os pacientes que participaram da pesquisa, em que foram abordados assuntos relacionados às medidas de cuidado integral com os pés enfatizando sua importância na prevenção de possíveis complicações.

O método de análise dos dados utilizado foi o de Análise de Conteúdo. De acordo com os aspectos éticos o estudo foi conduzido em conformidade com o Comitê de Ética e Pesquisa (CEP) e todos os pacientes assinaram o Termo de Consentimento Livre e Esclarecido (TCLE). Os pacientes tiveram autonomia sobre a participação de qualquer etapa da pesquisa. As informações obtidas foram analisadas em conjunto com as de outros pacientes, e não será divulgada a identificação de nenhum paciente. $\mathrm{O}$ estudo ao qual o projeto se vincula foi aprovado pelo CONSEPE local (049/2013).

\section{RESULTADOS}

A amostra foi do tipo aleatória, sendo critérios de inclusão ser portador de DM tipo 2 com tempo mínimo de diagnóstico de 10 anos. Excluídos os que haviam realizado cirurgias cardiovasculares no ano que antecedeu o estudo e indivíduos gestantes. O estudo contou com a participação de 113 indivíduos com idade média de 65,54 anos \pm 8,92, sendo 75,9\% da amostra composta por mulheres. Com relação a etnia, a maior parte da amostra foi composta por autodeclarados pardos, representando 50,9\%, seguida de pretos, amarelos e indígenas respec8tivamente $37,9,1,7 \%$ e $0,9 \%$. Quanto ao grau de escolaridade, $27 \%$ declararam-se analfabetos, $60 \%$ afirmaram ter estudado até o fundamental, 9,6\% nível médio e 3,4\%, nível superior. A renda mensal corresponde a menos de 1 salário mínimo para $19 \%$ dos indivíduos e de 1 a 3 salários para $81 \%$, que seria explicado pelo do alto custo econômico, gerado pelo tratamento e pela redução da capacidade de trabalho de indivíduos em idade produtiva (BRASILEIRO, JL 2005).

Hipertensão Arterial Sistêmica (HAS) estava presente em $87 \%$ da amostra, doença cardiovascular não hipertensiva em $21,8 \%$, dislipidemia em $60,2 \%$ e obesidade em $33,9 \%$. Do total da amostra 26,7\% fumavam. e 64,7\% dos indivíduos declararam-se sedentários (Tabela $1)$.

Tabela 1 - Caracteristicas clínicas da amostra

\begin{tabular}{l|c|c}
\hline VARIÁ $V E I S$ & N & \% \\
\hline FUMANTE & 31 & 26 \\
SEDENTARISMO & 75 & 64 \\
DOENÇAS CARD.* & 32 & 28 \\
HAS & 100 & 87 \\
OBESIDADE & 39 & 33
\end{tabular}




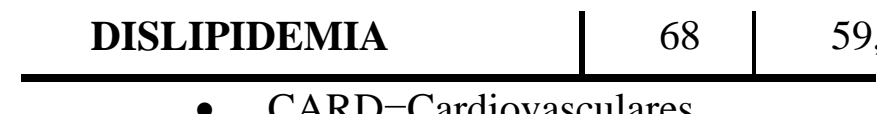

- $\quad$ CARD=Cardiovasculares.

Quanto a amputação, 5,6 \% tiveram membros amputados, representando ainda um número preocupante. Somente $20,2 \%$ utilizavam calçados de solado rígido, inadequados para a proteção do pé diabético, aumentando o risco de ocorrência de lesões. Mas, apenas 7,9\% apresentavam deformidades provocadas pelo tipo de calçado utilizado.

O Diabetes aumenta os níveis de açúcar no sangue, e o inadequado controle glicêmico provoca lesões progressivas das fibras somáticas (sensitivas e motoras) e autonômicas (FERREIRA, LT 2001). Como resultado, os diabéticos perdem a sensibilidade nos pés, deixando-os vulneráveis a ferimentos por quaisquer objetos, podendo chegar ao desenvolvimento de úlceras. Embora 96,5\% da amostra populacional saiba da importância do uso de calçados adequados e 94,7\% recebam orientações sobre sua comorbidade, apenas $28,1 \%$ utilizam desse tipo de calçado.

A respeito do conhecimento sobre a distribuição gratuita de calçados adequados, $11,4 \%$ disseram ter conhecimento dessa distribuição, 96,5\% consideram importante a distribuição gratuita de sapatos adequados ao portador de DM no entanto apenas $0,9 \%$ tinham acesso a esta distribuição. Da amostra, 51\% considera o uso de calçado adequado importante para prevenção de complicações nos pés, $28 \%$ acredita que proporcione uma melhora na caminhada, $19 \%$ considera importante devido ao maior conforto e apenas $1 \%$ acredita que a utilização de calçados adequados não promova benefícios.

Tabela 2- Análise do conhecimento do portador de Diabetes Mellitus a respeito da sua patologia e dos cuidados necessários.

\begin{tabular}{cr|r}
\hline \multicolumn{2}{l|}{ VARIÁVEIS } & \multicolumn{1}{c}{ N } \\
\hline Orientação sobre o DM na USF* & 5 & $4,4 \%$ \\
Não & 108 & $94,7 \%$ \\
Sim & & \\
Sabe distribuição gratuita de calçados & 100 & $87,7 \%$ \\
Não & 13 & $11,4 \%$ \\
Sim & & \\
Necessidade de distribuição gratuita & 3 & $2,6 \%$ \\
Não & 110 & $96,5 \%$ \\
Sim &
\end{tabular}

*USF= Unidade de Saúde da Família

\section{CONCLUSÕES}

Este estudo permitiu avaliar a freqüência dos fatores de riscos para amputação e a disponibilidade nas Unidades de Saúde de serviços relacionados a prevenção, terapia e reabilitação de indivíduos portadores de DM que sofreram amputação dos membros inferiores. Conclui-se portanto, que há elevada presença de fatores de risco para PD entre os indivíduos da amostra, que os mesmos estão cientes da importância do cuidado com os pés para prevenção de lesões e úlceras, no entanto, não a praticam e para que isso ocorra, é necessário implementar boas práticas educativas que transmitam informações atualizadas, claras e objetivas, buscando uma troca de informações entre a equipe multiprofissional, 
paciente e família assim como maior disponibilização de recursos para prevenção, cuidado e reabilitação dos portadores de DM e PD.

\section{REFERÊNCIAS}

1. Sociedade Brasileira de Diabetes. Diretrizes da Sociedade Brasileira de Diabetes. São Paulo: AC Farmacêutica, 2016.

2. International Diabetes Federation. IDF Diabetes atlas- $7^{\text {TH }}$ EDITION , 2017. <http://www.diabetesatlas.org/ > . Acesso em: 01 de Agosto de 2017.

3. Wild, S., et al. Global prevalence of diabetes: estimates for the year 200 and projections for 2030. Diabetes Care. 2004;5:1047-53

4. APELQVIST, J. et al. International Working Group on the Diabetic Foot (IWGDF) Editorial Board. Practical guidelines on the management and prevention of the diabetic foot: based up on the International Consensus on the Diabetic Foot (2007) prepared by the International Working Group on the Diabetic Foot. Diabetes Metab Res Rev. 2008;24 Suppl1:S181-7.

5. Brasileiro, JL Et al. Pé diabético: aspectos clínicos Jornal Vascular Brasileiro 2005, 4 (Março) : Consultado em: 18 de julho de 2017] Disponivel em $: \leq$ http://www.redalyc.org/articulo.0a?id=245020496004> ISSN 1677-5449

6. Ferreira, LT Et al. Diabetes melito: hiperglicemia crônica e suas complicações, Arquivos

Brasileiros de Ciências da Saúde, v.36, n. 3, p. 182-8, Set/Dez 2011

7. Glossary Guidance, International Working Group on the Diabetic Foot, 2007. Disponível em:< http://iwgdf.org/consensus/glossary//>. Acesso em: 17 de março de 2017. 